\title{
Optimization of a Capacitated Vehicle Routing Problem for Municipal Solid Waste Collection Using an Intelligence Hybrid Harmony Search Algorithm
}

\section{Wullapa Wongsinlatam}

Khon Kaen University https://orcid.org/0000-0002-7884-9093

Ayuwat Thanasate-angkool ( $\square$ ayuwat@kku.ac.th )

Khon Kaen University https://orcid.org/0000-0002-2419-6613

\section{Research}

Keywords: vehicle routing, supply chain network, mathematical modeling, metaheuristic optimization, waste management

Posted Date: August 13th, 2021

DOl: https://doi.org/10.21203/rs.3.rs-764365/v1

License: (c) (i) This work is licensed under a Creative Commons Attribution 4.0 International License.

Read Full License 


\title{
RESEARCH
}

\section{Optimization of a Capacitated Vehicle Routing Problem for Municipal Solid Waste Collection Using an Intelligence Hybrid Harmony Search Algorithm}

\author{
Wullapa Wongsinlatam ${ }^{1}$ and Ayuwat Thanasate-angkool ${ }^{1 *}$
}

\author{
${ }^{*}$ Correspondence: \\ ayuwat@kku.ac.th \\ ${ }^{1}$ Faculty of Interdisciplinary \\ Studies, Khon Kaen University, \\ Nong Khai Campus, Nong \\ Khai,43000, Thailand \\ Full list of author information is \\ available at the end of the article
}

\begin{abstract}
First part title: The study of sustainable management of municipal solid waste (MSW) collection has been increasing in recent years. However, the focus areas of research are mostly in the economics and environmental dimensions. This paper adds social aspects of MSW into considerations to accelerate more comprehensive decision making. The consideration on the capacity and fixed costs of vehicles, the distances from depot to the disposal facilities are determined in this paper. The environmental issues relating to fuel consumption, carbon emissions, and the evaluation of social impact from the penalty costs of imbalanced trip assignments are also determined to adopt a comprehensive solution to the capacitated vehicle routing problem (CVRP) model. Then, the optimization model of MSW collection is proposed to minimize management costs which compose of the fixed costs of vehicles, fuel consumption costs, carbon emissions costs, and penalty costs.
\end{abstract}

\section{Second part title:}

In this paper, two metaheuristic techniques are used to optimization the CVRP model in the MSW collection system. A new metaheuristic algorithm, called an intelligence hybrid harmony search algorithm (IHHS), is proposed in comparison with the standard harmony search (SHS) algorithm. The result shows that the IHHS algorithm can obtain the global optimal solution and can minimize the total objective function for the CVRP model.

Keywords: vehicle routing; supply chain network; mathematical modeling; metaheuristic optimization; waste management

\section{Introduction}

The increasing number of the world populations in recent years has accelerated concern over the sustainable management of municipal solid waste (MSW). MSW commonly refers to solid waste from residential, commercial, institutional sources, and hazardous waste [1-5]. The management of MSW generally includes collecting, transporting, processing, and disposing of waste [6] which requires a large amount of management costs. Thus, the MSW collection management organizations are faced with big challenges in planning the operation of the collection process. In addition, the campaign for sustainable development calls for the embracement of three fundamental sustainability parts: environmental protection, economic growth, and social equity which organizations are required to take into consideration [7]. 
In the matter of environmental protection, MSW is mostly concerned with the transportation network. The increasing number of carbon dioxide emissions are the most commonly assessed criteria of the environmental problem in MSW collection [8]. Due to the fact that the MSW collection process consumes energy and is the key driving force of carbon dioxide emission [1,9], it can be concluded that the MSW collection is in part responsible for public health and global warming in the cities [9]. Thus, for objective function in MSW collection, lower operating costs and carbon emissions have to be tackled, while social sustainability cannot be disregarded $[6,10]$. In the transportation network, vehicles at the depot start to collect a certain amount of waste, then the vehicles need to unload the waste at the disposal facilities (recycling facilities). However, even if a schedule has low operating and fixed vehicles costs, it cannot reach a good balancing trip to the recycling facilities, if each vehicle is not performing the same number of tasks [11].

The issue of the capacitated vehicle routing problem (CVRP) is one of the vehicle routing problem which is the NP-hard problem that is being researched around the world. The NP-hard problem is a complex problem which requires time consuming steps of calculation to reach a solution. The basic question in CVRP is the optimal route under the location constraints to find the shortest routes. Many exact and heuristic methods are studied and proposed to solve the CVRP [12]. Exact methods contain algorithms, such as branch and bound [13], branch and cut [14], and branch and price [15]. However, heuristic methods are more frequently used in large-scale CVRPs to reduce time and complexity.

Still, with CVRP, heuristic algorithms have limitations in its precision and require a long execution time [16]. Therefore, the movement toward metaheuristic algorithms have become more popular in recent years especially when incompleted information or limited computation capacity is a given [17]. The ability to find and develop the solution by using its own metaheuristic algorithms expand its the capacity to solve various problems, which include but are not limited to science, medical, agricultural, and engineering areas. Most of new metaheuristics are derived from the concepts of science and social science, such as particle swarm optimization (PSO) [18], ant colony optimization (ACO) [19-20], cuckoo search algorithm (CS) [21], firefly algorithm (FA) [22-24], and harmony search algorithm (HS) [25-28]. Furthermore, most of metaheuristic algorithms are developed from the prior ones, for example, Kuo et al. [29] combined hybrid particle swarm optimization (HPSO) with the genetic algorithm (GA) approach called the HPSOGA algorithm for solving capacitated vehicle routing problems with fuzzy demand (CVRPFD). Liu and He [30] adopted a clustering-based multiple ACO approach called the CMACS algorithm for solving vehicle routing problems with time windows. Recently, Yassen et al. used HS algorithm and variable neighborhood descent (VND) algorithms for solving a dynamic VRP with time windows [26]. While Maleki et al. used hybrid self-adaptive global best HS for solving VRP with time windows [27].

In summary, there is still limited research in the CVRP where the three factors of economy, environment, and society are being put into consideration. In addition, for sustainable development in MSW collection used in the CVRP model, previous studies rarely adopt hybrid metaheuristic algorithms. Considering the key characteristics and gaps of the learned literature, this paper creates a new metaheuristic 
algorithm based on a HS algorithm, and focuses on maximizing the comprehensive benefits which are economic, environmental, and social. The economic benefits being considered are the vehicles and their fuel consumption costs. The carbon dioxide emissions from transportation activities are assessed when considering the environmental factor, while the social factor in this research focuses on the number of trips to and from the disposal facilities.

In this paper, two metaheuristic techniques are used to compare the findings; a new metaheuristic algorithm, which is called, an intelligence hybrid harmony search (IHHS) algorithm, and the standard harmony search (SHS) algorithm. The rest of the paper proceeds as follows: Section 2 presents the mathematical model to find the CVRV and the objective function with constraints based on a detailed analysis of four optimization objectives. Section 3 presents two metaheuristic algorithms, and the comparison of the IHHS algorithm and the SHS algorithm in solving CVRP model. A case study of the real problem is also discussed in this section. Then, the computational results and discussion about the effectiveness of the proposed metaheuristic algorithms for solving CVRP model are provided in Section 4. Finally, Section 5 summarizes our conclusions with our future works.

\section{Mathematical model}

Problem Description

The municipal solid waste (MSW) collection problem uses the CVRP model to minimize the cost of management. The CVRP model defines a set of collection points by a homogenous set of vehicles of fixed capacity, each vehicle starts from one depot and returns to the same one empty [31]. Thus, when the vehicles reach a certain amount of waste collection, it needs to be unloaded at a disposal facility. However, due to the processing limit of disposal facilities, it is necessary to balance the workload of each trip as well. In this paper, the objective function of this model is to minimize the total costs of waste management by the arrangement of collection routes and the distribution of appropriate number of vehicles to the disposal facilities. The diagram of the MSW collection problem is shown Figure 1.

\section{Problem Assumptions}

The MSW collection problem is defined into five assumptions. First, this model assumes that there is only one depot from which all vehicles start at the same time, and return to the same depot eventually. Second, all of the vehicles start and end their trips with an empty load. Third, all vehicles have the same capacity limit of waste collection. Fourth, each of the collection points should be served once by one vehicle which are homogeneous with the same capacity loads. Finally, the vehicles may make multiple trips to collect the waste on their assigned route.

\section{Parameters and Variables}

In order to formulate a new mathematical model, sets, variable, and parameters must be defined. The symbol description is shown as follows: 
Figure 1 The diagram of the MSW collection

Sets

$G=$ Set of all the nodes in the network, $G=\{P, V\}$

$V=$ Set of vehicles, $\{k \mid k=1,2,3, \ldots, K\}$

$P=$ Set of collection points, $\{i \mid i=0,1,2,3, \ldots, R\}, 0$ is the depot

$S=$ Set of disposal facilities, $\{l \mid l=1,2,3, \ldots, D\}$

$M=$ Set of sub-paths, $\{n \mid n=1,2,3, \ldots, N\}$

\section{Decision Variables}

The decision variables of the CVRP model, $x_{i j k}, y_{i j}, z_{n k i}^{l}$, and $f_{n}^{l}$ are defined in binary number of 0 or 1 . Based on the details of four decision variables, the binary number of decision variables is shown as follows:

$$
\begin{aligned}
& x_{i j k}= \begin{cases}1 ; & \text { if vehicle } k \text { visits from point } i \text { to point } j \\
0 ; & \text { otherwise }\end{cases} \\
& y_{i j}= \begin{cases}1 ; & \text { if vehicle } k \text { visits from point } i \\
0 ; & \text { otherwise }\end{cases} \\
& z_{n k i}^{l}=\left\{\begin{aligned}
1 ; & \text { if sub-path } n \text { of vehicle } k \text { unloads waste at disposal facility } l \\
& \text { includes point } i \text { served by vehicle } k \\
0 ; & \text { otherwise }
\end{aligned}\right.
\end{aligned}
$$

\section{Parameters}

$Z_{i j k}=$ Carried load of vehicle $k$ visits from point $i$ to point $j$

$Z=$ Maximal load capacity of the vehicle

$g_{j}=$ Waste collection demand at the collection point $j$

$w_{l}=$ Workload limit of the disposal facility $l$

$d_{i j}=$ Transportation distance from point $i$ to point $j$

$C_{v}=$ Fixed costs per unit of vehicle

$C_{f}=$ Fuel consumption costs per unit

$C_{c}=$ Carbon emission costs per unit

$\mu=$ Fuel consumption rate when vehicle is at full load consumption rate

$\mu_{0}=$ Fuel consumption rate when vehicle is empty

$\rho=$ Conversion factor of carbon dioxide and fuel consumption

$\omega=$ Penalty cost of overload disposal facility per sub-path

\section{Objectives Function}

Vehicles' Fixed Costs

This paper assumes that each vehicle has at least one driver and one crew. Thus, the salaries costs for drivers and crews need to be considered in this method. $C_{V F}$ 
represents the vehicles' fixed costs for the CVRV model, it is shown as Equation (1).

$$
C_{V F}=C_{v} \cdot \sum_{k=1}^{K} \sum_{i=0}^{R+D} \sum_{j=0}^{R+D} x_{i j k}
$$

\section{Fuel Consumption Costs}

Many factors are the causes of fuel consumption of the vehicle, such as, the road condition, the vehicle characteristics, the running speed, etc. In this paper, $C_{F C}$ represents the fuel consumption costs in the CVRV model. It can be calculated as Equation (2).

$$
C_{F C}=C_{f} \cdot \sum_{k=1}^{K} \sum_{i=0}^{R+D} \sum_{j=0}^{R+D}\left(\mu+\frac{\mu-\mu_{0}}{Z} \cdot Z_{i j k}\right) \cdot d_{i j} \cdot x_{i j k}
$$

\section{Carbon Emission Costs}

The amount of carbon dioxide from fuel consumption is calculated into the carbon emissions of transportation. Therefore, $C_{C E}$ represents the carbon emissions costs of the CVRP model. The carbon emissions costs can be calculated as Equation (3).

$$
C_{C E}=C_{c} \cdot \rho \cdot \sum_{k=1}^{K} \sum_{i=0}^{R+D} \sum_{j=0}^{R+D} \sum_{n=1}^{N} \sum_{l=1}^{D}\left(\mu+\frac{\mu-\mu_{0}}{Z} \cdot Z_{i j k}\right) \cdot d_{i j} \cdot x_{i j k} \cdot Z_{n k i}^{l}
$$

\section{Penalty Costs}

The penalty costs are imposed on the disposal facility. A number of vehicles are assigned to the disposal facility, while each facility has limitations related to the capacity and the total number of collections. The processing capacity and penalty costs are calculated as Equation (4).

$$
C_{P C}=C_{c} \cdot \omega \cdot \sum_{n=1}^{N} \sum_{l=1}^{D} f_{n}^{l}
$$

\section{Mathematical model of the CVRP model}

Based on the detailed analysis, the new mathematical model of the CVRP model uses four optimization objectives with the constraints. The minimization of the CVRP model is shown as follows: 


$$
\begin{aligned}
& \text { Min TC }=C_{v} \cdot \sum_{k=1}^{K} \sum_{i=0}^{R+D} \sum_{j=0}^{R+D} x_{i j k} \\
& +\left(C_{f}+C_{c} \cdot \rho\right) \cdot \sum_{k=1}^{K} \sum_{i=0}^{R+D} \sum_{j=0}^{R+D} \sum_{n=1}^{N} \sum_{l=1}^{D}\left(\mu+\frac{\mu-\mu_{0}}{Z} \cdot Z_{i j k}\right) \cdot d_{i j} \cdot x_{i j k} \cdot Z_{n k i}^{l} \\
& +C_{c} \cdot \omega \cdot \sum_{n=1}^{N} \sum_{l=1}^{D} f_{n}^{l}
\end{aligned}
$$

Subject to:

$\sum_{j=1}^{R} Z_{0 j k}=0, \forall k \in V$

$\sum_{j=1}^{R} Z_{i j k} \leq Z, \forall k \in V$

$\sum_{k=1}^{K} y_{i k}=1, \forall i=1,2,3, \ldots, R$

$\sum_{i=0}^{R} \sum_{k=1}^{K} x_{i j k}=1, \forall j=1,2,3, \ldots, R$

$\sum_{i=0}^{R} \sum_{k=1}^{K} Z_{i j k}-\sum_{i=0}^{R} \sum_{k=1}^{K} Z_{i j k}=g_{j}, \forall j=1,2,3, \ldots, R$

$x_{l j k} \leq z_{n k i}^{l}, \forall k \in V, \forall l \in S, \forall n \in M, \forall i, j=1,2,3, \ldots, R+D$

$$
\sum_{i=0}^{R} \sum_{k=1}^{K} x_{i 0 k}=1
$$

$$
d_{i j} \leq d_{j i}, \forall i, j=1,2,3, \ldots, R+D
$$

In Equation (5), the objective of the model is to minimize the management costs of waste collection (Min $T C=C_{V F}+C_{F C}+C_{C E}+C_{P C}$ ). The goal of the model 
is to use four defined costs: fixed costs of vehicles, fuel consumption costs, carbon emission costs, and penalty costs, respectively. The constraints of the objective function are; first, in constraint (6), all vehicles start from depot without waste. Second, in constraint (7), each vehicle cannot exceed its own load capacity. Third, in constraints (8) and (9), each collecting points are served by one vehicle and all points must be served. Fourth, in constraint (10), the vehicle must empty at the collection point. Fifth, in constraint (11), when vehicle $k$ visits the disposal facility $l$ for dumping. Sixth, in constraint (12), all vehicles return to the depot eventually. And finally, in equation (13), the distance between two points are the same in both directions.

\section{Metaheuristic Methods}

Focusing on economic, environment, and social benefits, this paper takes into consideration carbon emissions generated from fuel consumption, and the penalty costs which are derived from imbalanced trip assignments to the disposal facilities. Thus, the objective function of the CVRP model is proposed to decrease management costs which are the fixed costs of vehicles, fuel consumption costs, carbon emissions costs, and penalty costs. In this paper, two metaheuristic techniques are used; a new metaheuristic algorithm called an intelligence hybrid harmony search (IHHS) algorithm is compared with standard harmony search (SHS) algorithm.

\section{Standard Harmony Search}

Standard Harmony Search (SHS) algorithm is a population-based metaheuristic optimization. It is defined to represent the music improvisation where the musicians improvise the harmony. An orchestra performance consists of a saxophone, a double bass and a guitar. The music instruments can have a very high pitch range. For example, the instrument pitch for saxophone is Do, Re, Mi, the double bass is Mi, Fa, $\mathrm{Sol}$, and the guitar is Sol, La, Si. If the saxophone is randomly chosen Re, the double bass is randomly chosen $\mathrm{Fa}$, and the guitar is La, respectively. If this new harmony is better than old harmony (worst) in the harmony memory. Next, the worst harmony will be replaced by the new harmony. Thus, the SHS algorithm has three options, first, play any pitch from the memory, second, play something similar to others in their memory, and finally, randomizing the notes. This algorithm is repeated until a perfect harmony is found and until a stopping criterion for termination is reached or the optimal solution is found. The SHS algorithm for optimal solutions is linked with the analogy between the music improvisation and the optimization problem in the part of the searching of a perfect state of harmony [25]. The concept of the SHS algorithm is shown in Fig. 2. Below are the steps design of the SHS algorithm.

\section{Step 1: Problem formulation}

The SHS algorithm must be formulated into an optimization problem with the objective function and constraint. The SHS algorithm searches the optimal solution. The vector $x=\left(x_{1}, x_{2}, \ldots, x_{n}\right)$ that is maximized or minimized for the objective function. If the decision variables have discrete values, the set of possible values is given by $x_{i} \in X_{i}=\left\{x_{i}(1), x_{i}(2), \ldots, x_{i}(V)\right\}$ where $V_{i}$ is the number of different values in the definition space for variable $i$. If the variables have continuous values, 
the set of possible values is given by $x_{i}^{L} \leq x_{i} \leq x_{i}^{U}$ where $L$ is the lower bound and $U$ is the upper bound, respectively.

\section{Step 2: Parameter setting}

The parameters of the SHS algorithm must be set with values. The parameters setting used in the SHS algorithm are harmony memory considering rate (HMCR), pitch adjusting rate (PAR), harmony memory size (HMS), stopping criterion and bandwidth (bw) that operate altogether with PAR in pitch adjustment. HMCR is important to ensure that good solutions are considered as element of new solutions. Therefore, in order to use the memory effectively, the value of HMCR should be in between 0.7 and 0.95 (Yang, 2010). Given the limitation in exploration to a single portion of the search space, a small value of PAR together with narrow value of bw can cause the convergence of the SHS algorithm to be slow. However, a high value of PAR can cause solutions to disperse around a few potential optimal as in random search. Thus, the value of PAR is around 0.1 and 0.5 and the bw generally bounded between $1 \%$ and $10 \%$ of all the range of variable values (Yang, 2010).

Step 3: Initialize the memory

To create the initial memory, several solutions are generated randomly and the number of solutions should be at least equal to the HMS. The matrix of HM can be described as the following matrix:

$$
H M=\left(\begin{array}{cccc|c}
x_{1}^{1} & x_{2}^{1} & \cdots & x_{n}^{1} & f\left(x^{1}\right) \\
x_{1}^{2} & x_{2}^{2} & \cdots & x_{n}^{2} & f\left(x^{2}\right) \\
\vdots & \vdots & \ddots & \vdots & \vdots \\
x_{1}^{H M S} & x_{2}^{H M S} & \cdots & x_{n}^{H M S} & f\left(x^{H M S}\right)
\end{array}\right),
$$

where $x_{i}^{j}$ is a decision variable for $i=1,2,3, \ldots, n$ and $j=1,2,3, \ldots, H M S$ and $f\left(x^{j}\right)$ is fitness function or objective function of the problem for $j=1,2,3, \ldots, H M S$.

\section{Step 4: Improvisation}

The process of improvisation in SHS algorithm has three processes. The first process is random selection, when SHS determine the new solution $x^{\text {new }}$, it randomly chooses a value from the range of all possible values $x_{i}^{L} \leq x_{i} \leq x_{i}^{U}$ with a probability $(1-$ HMCR $)$. The second process is memory consideration, when the probability equals to HMCR, the new solution, $x^{\text {new }}$ is chosen randomly from the HM. The random number may be calculated using a uniform distribution $U(0,1)$. The final process is pitch adjustment, after the value of new solution has been randomly chosen from the HM, it may be adjusted to neighbouring values with probability PAR. For discrete variable, $x^{\text {new }}=x_{i}(k+n)$ where $n \in\{-1,1\}$ and $k$ is the position of a chosen solution in HM. For continuous variable, the new solution vector will be $x^{\text {new }}=x_{i}+\triangle$ where $\triangle=U(-1,1) \times b w(i)$.

Step 5: Memory update

If the new solution, $x^{\text {new }}$ is better than the worst solution in HM in terms of the objective function value, the new solution will replace the worst solution. The HM will be updated and sorted according to the value of the objective function.

\section{Step 6: Termination}


Figure 2 Analogy between music improvisation and optimization problem (Geem et al., 2001)

If the SHS algorithm meets the stopping criterion such as the maximum iteration or maximum execution time, the process will be terminated.

\section{A new metaheuristic algorithm}

In this paper, a new metaheuristic algorithm to solve the problem is an intelligence hybrid harmony search (IHHS) algorithm, it is proposed and described in the following steps.

\section{Step 1: Problem formulation}

The CVRP model with one depot, $R$ is the collection point, $D$ is the disposal facilities, and $N$ is the sub-paths consists of $2 R+N+1$ dimensional. We focuses on four parts: collection points, depot, disposal facilities, and encodings of sub-paths. The creation of the first new process of IHHS algorithm is called Inteligent-1, it defines encoding of sub-path, and $R$ is direction. The $m$ is the minimum number of sub-path. The value of each direction of the sub-path number to each collection point is randomly selected from the natural number of 1 to $n$. The creation of the second new process of IHHS algorithm is called Inteligent-2, it defines the encoding of the collection point, that is also defines $R$ directions. The value of directions represent the order of all collection points in each sub-path and is randomly chosen from 1 to $R$ (natural number). There is one correlation in Inteligent- 1 and Inteligent2 , the collection points in Inteligent- 2 corresponding to the directions have the same value as to the Inteligent-1. However, while the sub-path is calculated by the total demand divided by the vehicle capacity, still, the load of the vehicle have the capacity limitation. Thus, if the load of the sub-path exceed the vehicle capacity limit, a new sub-path appears and the remaining collection points will be moved to the new sub-path. Next, the number of sub-path will be added up to a new $N$. Inteligent-3 is encoding of disposal facility, that defines $R$ directions. The value of each direction is represented in the initial disposal facility corresponding to each subpath which is randomly selected from $R+1$ to $R+D$ (natural number). Inteligent- 4 is encoding of the depot, that is defined only one direction. Hence, all vehicles start from the same depot, encode the one depot as $R+D+1$ (natural number). Finally, the total length of coding is $2 R+N+1$. For one depot capacity limit, the collection points are clustered. The assigned vehicle visits from point $i$ to point $j$ and the distance is calculated using the Euclidean distance formula.

\section{Step 2: Parameter setting}

In IHHS algorithm, the parameter used in solving the CVRP model and the HM is presenting the population of the solutions. The HMS is setting to 300 , that is presenting the size of the solutions in that population. The new HMS for IHHS algorithm is using 20 as the size of the newly generated solutions. The HMCR is setting to 0.83 , and the PAR is setting to 0.25 , respectively.

\section{Step 3: Initialize the memory}

All the solution vectors in the population randomly create the initial HM, and the number of solutions are equal to HMS. In IHHS algorithm, we implemented algorithm to generate a solution vector in the initial population. The steps of initialize 
the memory for IHHS algorithm are described as follows, step 1: When all vehicles start at one depot with the calculated saving, all vehicle visit collection points, visit disposal facility, and visit the depot again. Step 2: Sort the saving in descending order. Step 3: Start to merge the route for the most saving, the following process should be fulfilled to merge the route, and two nodes can be combined into a same route if the total demand does not exceed the vehicle limit capacity.

\section{Step 4: Improvisation}

The several solutions of the IHHS algorithm are produced to create a new HM. The size of the new HM is less than the HMS. The following processes are conducted: The first process of improvisation is called random selection. The new solution vector is randomly produced to the permutation of the set collection points with probability of (1-HMCR). Next, second process of improvisation is called memory consideration. The roulette wheel selection is used to select the new solution vector $x^{\text {new }}$ in the HM, that is compared to a probability with equals to HMCR. The absolute fitness values of any solution compared to the absolute fitness values of other solution in HM. The selection probability $p_{i}$ and cumulative probability $q_{j}$ for the solution $i$ and $j$ is calculated by Equation (14) and (15),

$$
\begin{aligned}
p_{i} & =\frac{T C_{i}}{\sum_{i=1}^{H M S} T C_{i}}, \\
q_{j} & =\sum_{i=1}^{j} p_{i},
\end{aligned}
$$

where, $T C_{i}$ is the objective function of the CVRP model. The selection of solution is generated by the random number of 0 and 1 . If the random number is within the range of $q_{j}$ and $q_{j+1}$ for the cumulative probability, the solution $j+1$ is chosen as the next solution. In optimization problem, the formulation of calculation $p_{i}$ is different and the fitness values in the HM are sorted. Next, the fitness function should be inverted into $1 / T C_{i}$. Next, the process of improvisation is called pitch adjustment. The first of new solution is produced, the adjustment of neighbouring structure in a form of local optimization is applied to enhance the exploration and exploitation capabilities of the algorithm. In IHHS algorithm, the local optimization of swapping, insertion and relocation are used based on the values of PAR. All vehicles start from the depot to the collection point, then to disposal facility, and return to depot. There are two techniques for swapping which are the swapping of two collection points within the depot and between the disposal facilities. For relocation, a collection point will be relocated from one depot to another disposal facility.

Step 5: Memory update In IHHS algorithm, the HM and new HM are combined and sorted according to the value of the objective function for CVRP model. Then, the best solutions with the size of HMS are stored, and are used for the next iteration.

\section{Step 6: Termination}

The final step of the IHHS algorithm is setting iterations for stopping criteria. 
Table 1 The decoding example and the solution representation

\begin{tabular}{lllllllllll}
\hline \multicolumn{10}{c}{ Decoding example } \\
\hline Part 1 & 1 & 1 & 3 & 2 & 3 & 2 & 1 & 1 & 4 & 2 \\
Part 2 & 3 & 6 & 5 & 1 & 8 & 4 & 2 & 10 & 7 & 9 \\
Part 3 & 11 & 13 & 11 & 12 & & & & \\
Part 4 & 15 & & \\
\multicolumn{8}{c}{ Solution representation for vehicle routes } \\
\hline Solution 1 & $15 \rightarrow 3 \rightarrow 6 \rightarrow 2 \rightarrow 10 \rightarrow 11 \rightarrow 15$ \\
Solution 2 & \multicolumn{1}{c}{$15 \rightarrow 1 \rightarrow 4 \rightarrow 9 \rightarrow 13 \rightarrow 15$} \\
Solution 3 & $15 \rightarrow 5 \rightarrow 8 \rightarrow 11 \rightarrow 15$ \\
Solution 4 & $15 \rightarrow 7 \rightarrow 12 \rightarrow 15$ \\
\hline
\end{tabular}

Table 2 The data of the CVRP model

\begin{tabular}{cccccc}
\hline Instance & Collection Point & Disposal Facility & Vehicle & Workload Limit & Capacity \\
\hline p01 & 50 & 3 & 16 & 4 & 80 \\
p03 & 70 & 4 & 15 & 3 & 140 \\
p06 & 100 & 2 & 18 & 6 & 100 \\
p07 & 100 & 3 & 16 & 5 & 100 \\
p15 & 160 & 3 & 20 & 5 & 60 \\
\hline
\end{tabular}

\section{Experimental Results and Discussion}

The proposed CVRP model and the metaheuristics optimization are verified by testing benchmark data with difference sizes of collection points and disposal facilities. In this paper, all of datasets are derived from the VRP Web (http://www.bernabe.dorronsoro.es/vrp/). The work station used for carrying out the result is equipped with $3.00 \mathrm{GHz}$ of processor, 4.00 GB of RAM, 64-bit operating system, x-64 based processor of system type and the system used Microsoft Windows 10. The program that is used in this research is MATLAB R2019b which is software for numerical computation.

The experimental design of the CVRP model

The benchmark datasets of CVRP model, ten collection points are defined into number 1 to 4 , four disposal facilities are defined as number 11 to 14 , and one depot is defined as number 15. Therefore, the sub-path number is $N=4$ and is part 1 to 4 . The example and the solution for the problem are shown in Table 1.

In this paper, the first start is the depot, and the other stops are affirmed as disposal facilities. Five instances of routes are chosen. Each instance contains the number of collection points, one depot, disposal facilities, number of vehicles with the capacity, and workload limit of each disposal facility. The workload limit means the upper limit of sub-paths for each disposal facility and each disposal facility has the same limit. The number of vehicles remains the same as in the initial instance. We used one depot for all the test instances while the data of the test instances are used in the CVRP model as shown in Table 2.

Table 3 shows the computational results of SHS and IHHS algorithm including the number of sub-paths and distances. The optimization rate was also calculated to highlight the performance of IHHS algorithm.

One can obviously see that the number of sub-paths and the distance calculated by IHHS algorithm is better compared with the results of the SHS. The proposed IHHS algorithm has a great performance in improving the quality of solutions. 
Table 3 Computational results of the SHS and IHHS algorithm

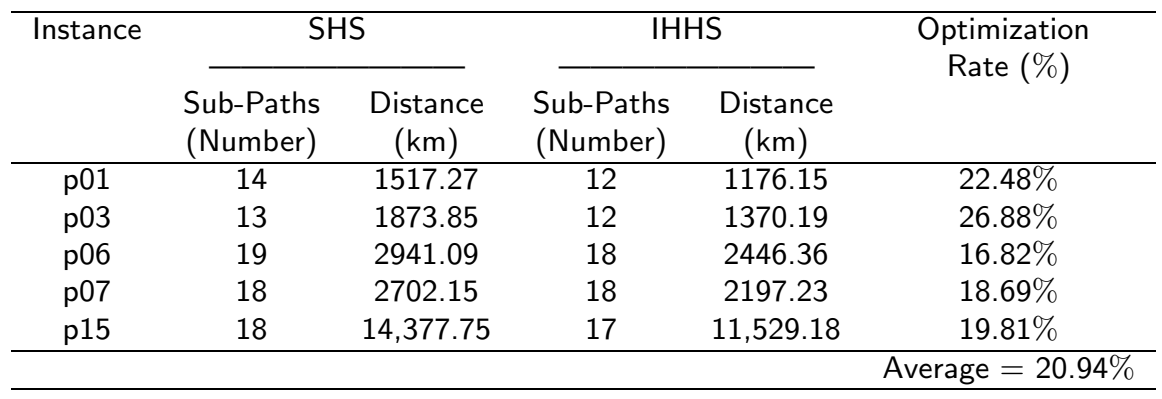

Table 4 Positions and information for MSW collection

\begin{tabular}{ccc}
\hline Depot & X Coordinate & Y Coordinate \\
\hline 1 & 30 & 40 \\
\hline Disposal Facilities & X Coordinate & Y Coordinate \\
\hline 1 & 20 & 20 \\
2 & 50 & 30 \\
3 & 60 & 50 \\
4 & 36 & 16 \\
5 & 42 & 57 \\
6 & 8 & 52 \\
\hline
\end{tabular}

The initial number of disposal facilities in the test instance p01 is too small to be tested. Thus, other three positions from collection points are chosen to be the new disposal facilities. Therefore, the numbers of disposal facilities and collection points change from the original 3 and 50 to 6 and 47, respectively. The process determined positions of the disposal facilities, the information about depot, and the position of the depot. Next, setting the number of vehicles (16 vehicles), and the maximal load capacity of the vehicle (80 maximal weight/t) are shown in Table 4 . To show the improvement in realistic scenario, the dataset p01 is set for 5 working days in a week and waste loads are in correspondence with the original data of p01. For illustration, the Monday positions and waste loads of collection points are shown in Table 5.

In order to obtain the results, a number is given as the limit for each disposal facility. The given number is greater or equal to the average number of sub-paths that is assigned to each disposal facility. The proposed algorithm is then set for 5 working days in a week. If a facility is assigned over limit collection trips, the given limit is decreased. Table 6 presents the upper limits for all 6 disposal facilities, where $W_{l}$ represents the given limit for disposal facility $l$.

The parameters related to the CVRP model of the IHHS algorithm, the fixed costs of vehicle per unit $C_{v}$ is $46.30 \mathrm{USD}$, the costs of fuel consumption per unit $C_{f}$ is 1.08 $\mathrm{USD} / \mathrm{L}$, the costs of carbon emission per unit $C_{c}$ is $0.09 \mathrm{USD} / \mathrm{kg}$, fuel consumption rate when vehicle is full load consumption rate $\mu$ is $0.38 \mathrm{~L} / \mathrm{km}$, fuel consumption rate when vehicle is empty $\mu_{0}$ is $0.17 \mathrm{~L} / \mathrm{km}$, conversion factor for carbon dioxide and fuel consumption $\rho$ is $2.32 \mathrm{~kg} / \mathrm{L}$, and the penalty value $\omega$ is set to half of the fixed costs of vehicle per unit which is 23.15 USD.

The experimental results of the CVRP model

The minimization of economic costs, carbon emissions, and penalty costs were included in to the experiment of the CVRP model. In addition, sampling variance is 
Table 5 Position and waste load on Monday

\begin{tabular}{cllllllllll}
\hline CollectionPoints & 1 & 2 & 3 & 4 & 5 & 6 & 7 & 8 & 9 & 10 \\
\hline $\mathrm{X}$ & 37 & 49 & 52 & 20 & 40 & 21 & 17 & 31 & 52 & 51 \\
$\mathrm{Y}$ & 52 & 49 & 64 & 26 & 30 & 47 & 63 & 62 & 33 & 21 \\
Waste Load/t & 7 & 30 & 16 & 9 & 21 & 15 & 19 & 23 & 11 & 5 \\
\hline CollectionPoints & 11 & 12 & 13 & 14 & 15 & 16 & 17 & 18 & 19 & 20 \\
\hline $\mathrm{X}$ & 42 & 31 & 5 & 12 & 52 & 27 & 17 & 13 & 57 & 62 \\
$\mathrm{Y}$ & 41 & 32 & 25 & 42 & 41 & 23 & 33 & 13 & 58 & 42 \\
Waste Load/t & 19 & 29 & 23 & 21 & 15 & 3 & 41 & 9 & 28 & 8 \\
\hline CollectionPoints & 21 & 22 & 23 & 24 & 25 & 26 & 27 & 28 & 29 & 30 \\
\hline $\mathrm{X}$ & 16 & 7 & 27 & 30 & 43 & 58 & 58 & 37 & 38 & 46 \\
$\mathrm{Y}$ & 57 & 38 & 68 & 48 & 67 & 48 & 27 & 69 & 46 & 10 \\
Waste Load/t & 16 & 28 & 7 & 15 & 14 & 6 & 19 & 11 & 12 & 23 \\
\hline CollectionPoints & 31 & 32 & 33 & 34 & 35 & 36 & 37 & 38 & 39 & 40 \\
\hline $\mathrm{X}$ & 61 & 62 & 63 & 32 & 45 & 59 & 5 & 10 & 21 & 5 \\
$\mathrm{Y}$ & 33 & 63 & 69 & 22 & 35 & 15 & 6 & 17 & 10 & 64 \\
Waste Load/t & 26 & 17 & 6 & 9 & 15 & 14 & 7 & 27 & 13 & 11 \\
\hline CollectionPoints & 41 & 42 & 43 & 44 & 45 & 46 & 47 & & & \\
\hline $\mathrm{X}$ & 30 & 39 & 32 & 25 & 25 & 48 & 56 & & & \\
Y & 15 & 10 & 39 & 32 & 55 & 28 & 37 & & & \\
Waste Load/t & 16 & 10 & 5 & 25 & 17 & 18 & 10 & & &
\end{tabular}

Table 6 Upper limits for disposal facilities

\begin{tabular}{ccccccc}
\hline Day & \multicolumn{9}{c}{ Upper Limits for } & Number of Sub-Paths \\
& $W_{1}$ & $W_{2}$ & $W_{3}$ & $W_{4}$ & $W_{5}$ & $W_{6}$ \\
\hline Monday & 2 & 2 & 2 & 2 & 2 & 2 \\
Tuesday & 2 & 2 & 2 & 2 & 2 & 2 \\
Wednesday & 3 & 3 & 3 & 3 & 3 & 3 \\
Thursday & 3 & 3 & 3 & 3 & 3 & 3 \\
Friday & 4 & 4 & 4 & 4 & 4 & 4 \\
\hline
\end{tabular}

Table 7 Results of the SHS algorithm

\begin{tabular}{|c|c|c|c|c|c|c|c|c|c|c|c|}
\hline \multirow[t]{2}{*}{ Day } & \multirow[t]{2}{*}{ Sub-Paths } & \multirow{2}{*}{$\begin{array}{c}\text { Distance } \\
(\mathrm{km})\end{array}$} & \multirow{2}{*}{$\begin{array}{c}\text { Carbon } \\
\text { Emissions } \\
(\mathrm{kg})\end{array}$} & \multirow{2}{*}{$\begin{array}{c}\text { Operational } \\
\text { Costs } \\
\text { (USD) }\end{array}$} & \multicolumn{6}{|c|}{ Sub-Path of Disposal Facilities } & \multirow{2}{*}{$\begin{array}{l}\text { Sampling } \\
\text { variance }\end{array}$} \\
\hline & & & & & $S_{1}$ & $S_{2}$ & $S_{3}$ & $S_{4}$ & $S_{5}$ & $S_{6}$ & \\
\hline Monday & 12 & 1136.57 & 398.42 & 909.42 & 2 & 2 & 2 & 2 & 2 & 2 & 2.01 \\
\hline Tuesday & 13 & 1118.68 & 398.54 & 955.95 & 2 & 3 & 2 & 2 & 2 & 2 & 2.98 \\
\hline Wednesday & 15 & 1207.65 & 418.59 & 1066.53 & 3 & 3 & 1 & 2 & 3 & 3 & 2.31 \\
\hline Thursday & 18 & 1354.07 & 474.83 & 1162.68 & 3 & 3 & 3 & 3 & 3 & 3 & 3.31 \\
\hline Friday & 21 & 1368.75 & 453.57 & 1143.84 & 4 & 4 & 3 & 2 & 4 & 4 & 2.71 \\
\hline
\end{tabular}

Table 8 Results of the IHHS algorithm

\begin{tabular}{|c|c|c|c|c|c|c|c|c|c|c|c|}
\hline \multirow[t]{2}{*}{ Day } & \multirow[t]{2}{*}{ Sub-Paths } & \multirow{2}{*}{$\begin{array}{c}\text { Distance } \\
(\mathrm{km})\end{array}$} & \multirow{2}{*}{$\begin{array}{c}\text { Carbon } \\
\text { Emissions } \\
(\mathrm{kg})\end{array}$} & \multirow{2}{*}{$\begin{array}{c}\text { Operational } \\
\text { Costs } \\
\text { (USD) }\end{array}$} & \multicolumn{6}{|c|}{ Sub-Path of Disposal Facilities } & \multirow{2}{*}{$\begin{array}{c}\text { Sampling } \\
\text { variance }\end{array}$} \\
\hline & & & & & $S_{1}$ & $S_{2}$ & $S_{3}$ & $S_{4}$ & $S_{5}$ & $S_{6}$ & \\
\hline Monday & 12 & 1023.52 & 351.48 & 867.90 & 3 & 4 & 0 & 1 & 2 & 2 & 0.00 \\
\hline Tuesday & 13 & 1039.37 & 364.45 & 925.80 & 4 & 3 & 0 & 1 & 4 & 1 & 0.17 \\
\hline Wednesday & 15 & 1105.72 & 372.02 & 1025.24 & 4 & 4 & 0 & 2 & 3 & 2 & 0.70 \\
\hline Thursday & 18 & 1222.07 & 421.24 & 1115.26 & 4 & 5 & 0 & 2 & 3 & 4 & 0.00 \\
\hline Friday & 21 & 1291.01 & 425.21 & 1118.48 & 5 & 5 & 2 & 1 & 4 & 4 & 0.70 \\
\hline
\end{tabular}

assigned to each disposal facility to observe the effectiveness of balancing. Therefore, a balanced schedule with the smallest sampling variance is preferred, even with slightly higher costs. The detailed results of two metaheuristic algorithms for the CVRP model from Monday to Friday are presented in Tables 7-8. 
Table 9 Detailed collection routes of the IHHS algorithm for the CVRP model

\begin{tabular}{|c|c|}
\hline Day & Collection Routes form Monday to Friday \\
\hline Monday & $\begin{array}{c}6 \rightarrow 17 \rightarrow 4 \rightarrow S_{1}, 42 \rightarrow 39 \rightarrow 37 \rightarrow 18 \rightarrow S_{1}, \\
16 \rightarrow 34 \rightarrow 12 \rightarrow 5 \rightarrow 46 \rightarrow S_{2}, 3 \rightarrow 19 \rightarrow 47 \rightarrow 27 \rightarrow 10 \rightarrow S_{2}, \\
33 \rightarrow 32 \rightarrow 20 \rightarrow 31 \rightarrow 9 \rightarrow S_{3}, 24 \rightarrow 2 \rightarrow S_{3}, \\
43 \rightarrow 35 \rightarrow 11 \rightarrow 29 \rightarrow 1 \rightarrow S_{4}, 44 \rightarrow 41 \rightarrow 30 \rightarrow 36 \rightarrow S_{4}, \\
15 \rightarrow 26 \rightarrow 25 \rightarrow 28 \rightarrow 23 \rightarrow 8 \rightarrow S_{5}, 22 \rightarrow 14 \rightarrow S_{5}, \\
\quad 13 \rightarrow 38 \rightarrow S_{6}, 45 \rightarrow 21 \rightarrow 7 \rightarrow 40 \rightarrow S_{6},\end{array}$ \\
\hline Tuesday & $\begin{array}{c}44 \rightarrow 17 \rightarrow S_{1}, 13 \rightarrow 38 \rightarrow S_{1} \\
5 \rightarrow 46 \rightarrow S_{2}, 1 \rightarrow 34 \rightarrow 10 \rightarrow 36 \rightarrow 27 \rightarrow 9 \rightarrow S_{2} \\
33 \rightarrow 32 \rightarrow 26 \rightarrow 31 \rightarrow S_{3}, 6 \rightarrow 45 \rightarrow 8 \rightarrow 28 \rightarrow S_{3} \\
19 \rightarrow S_{3}, 12 \rightarrow 42 \rightarrow 30 \rightarrow S_{4}, 14 \rightarrow 22 \rightarrow 4 \rightarrow S_{4} \\
35 \rightarrow 47 \rightarrow 15 \rightarrow 2 \rightarrow S_{5}, 43 \rightarrow 11 \rightarrow 29 \rightarrow 3 \rightarrow 25 \rightarrow S_{5} \\
16 \rightarrow 41 \rightarrow S_{6}, 39 \rightarrow 37 \rightarrow 18 \rightarrow S_{6} \\
24 \rightarrow 23 \rightarrow 7 \rightarrow 21 \rightarrow 40 \rightarrow S_{6}\end{array}$ \\
\hline Wednesday & $\begin{array}{l}5 \rightarrow S_{1}, 22 \rightarrow 13 \rightarrow 18 \rightarrow S_{1}, 12 \rightarrow 34 \rightarrow 16 \rightarrow 4 \rightarrow S_{1} \\
\quad 20 \rightarrow 31 \rightarrow 27 \rightarrow S_{2}, 35 \rightarrow 15 \rightarrow 47 \rightarrow S_{2} \\
36 \rightarrow 10 \rightarrow 46 \rightarrow 9 \rightarrow S_{2}, 24 \rightarrow 8 \rightarrow 23 \rightarrow 28 \rightarrow S_{3} \\
39 \rightarrow S_{3}, 37 \rightarrow 38 \rightarrow S_{4}, 30 \rightarrow 42 \rightarrow 41 \rightarrow S_{4} \\
32 \rightarrow 33 \rightarrow 3 \rightarrow 25 \rightarrow S_{5}, 14 \rightarrow 6 \rightarrow 21 \rightarrow S_{5} \\
\quad 19 \rightarrow 26 \rightarrow 2 \rightarrow S_{5}, 45 \rightarrow 7 \rightarrow 40 \rightarrow S_{6} \\
\quad 43 \rightarrow 11 \rightarrow 29 \rightarrow 1 \rightarrow S_{6}, 44 \rightarrow 17 \rightarrow S_{6}\end{array}$ \\
\hline Thursday & $\begin{array}{c}22 \rightarrow S_{1}, 14 \rightarrow S_{1}, 41 \rightarrow 39 \rightarrow 18 \rightarrow S_{1} \rightarrow 4 \rightarrow S_{1} \\
12 \rightarrow 16 \rightarrow 46 \rightarrow S_{2}, 11 \rightarrow 35 \rightarrow 9 \rightarrow S_{2}, \\
36 \rightarrow 10 \rightarrow 31 \rightarrow S_{2}, 19 \rightarrow 32 \rightarrow 33 \rightarrow S_{3} \\
45 \rightarrow S_{3}, 23 \rightarrow 28 \rightarrow S_{3}, 5 \rightarrow 47 \rightarrow 26 \rightarrow 20 \rightarrow S_{3} \\
43 \rightarrow 34 \rightarrow 30 \rightarrow 42 \rightarrow S_{4}, 15 \rightarrow 27 \rightarrow S_{4} \\
\quad 2 \rightarrow 1 \rightarrow S_{4}, 7 \rightarrow 40 \rightarrow S_{5}, 6 \rightarrow S_{5} \\
\quad 29 \rightarrow 3 \rightarrow 25 \rightarrow S_{5}, 44 \rightarrow 17 \rightarrow S_{6} \\
13 \rightarrow 37 \rightarrow 38 \rightarrow S_{6}, 24 \rightarrow 8 \rightarrow 21 \rightarrow S_{6}\end{array}$ \\
\hline Friday & $\begin{array}{c}39 \rightarrow 41 \rightarrow 34 \rightarrow 16 \rightarrow S_{1}, \rightarrow 17 \rightarrow S_{1} \rightarrow 13 \rightarrow 38 \rightarrow S_{1} \\
37 \rightarrow 18 \rightarrow S_{1}, 10 \rightarrow 46 \rightarrow 5 \rightarrow S_{2}, 30 \rightarrow 42 \rightarrow 36 \rightarrow S_{2} \\
27 \rightarrow S_{2}, 47 \rightarrow 31 \rightarrow 9 \rightarrow S_{2}, 20 \rightarrow 19 \rightarrow S_{3}, 15 \rightarrow 26 \rightarrow S_{3} \\
32 \rightarrow 33 \rightarrow 3 \rightarrow S_{3}, 43 \rightarrow 24 \rightarrow 1 \rightarrow S_{4}, 4 \rightarrow 12 \rightarrow S_{4}, 2 \rightarrow S_{5} \\
25 \rightarrow 28 \rightarrow S_{5}, 11 \rightarrow 29 \rightarrow 35 \rightarrow S_{5} \\
40 \rightarrow S_{5}, 44 \rightarrow 6 \rightarrow S_{6}, 22 \rightarrow 14 \rightarrow S_{6} \\
21 \rightarrow 7 \rightarrow S_{6}, 45 \rightarrow 23 \rightarrow 8 \rightarrow S_{6}\end{array}$ \\
\hline
\end{tabular}

From the detailed results in Tables 7-8, we can observe the following: First, when minimized penalty costs are added to the objective function in CVRP model, the distance of vehicles $(\mathrm{km})$, carbon emissions $(\mathrm{kg})$, and operational costs (USD) are less than the values for SHS algorithm every single day. Therefore, the values of sampling variance obtained by CVRP model for the IHHS algorithm are smaller than the values for SHS algorithm. If the sampling variance is very small, it means that the solution obtain is good. After accumulating from Monday to Friday, the sampling variance for the IHHS algorithm are smaller than the values for SHS algorithm. The sampling variance of the IHHS algorithm and the best known solution are less in all problems. The best detailed collection routes of the IHHS algorithm for the CVRP model from Monday to Friday is presented in Tables 9.

\section{Conclusions}

This research focuses in optimising the MSW collection problem in the CVRP model. The objective is to minimize economic costs, carbon emissions, and penalty 
costs by two metaheuristic techniques which are SHS and IHHS algorithms. The workstation used for carrying out the result is equipped with MATLAB. The research compares the result of performances to find the best choice in developing the management model. The result shows that the IHHS algorithm can obtain the global optimal solution, has a faster convergence and can minimize the total objective function for the CVRP model. In the future work, it will be useful to improve metaheuristic optimization for the fast computing and defining parameter optimization for a new set of the MSW collection problem.

\section{Appendix}

$\begin{array}{ll}\text { MSW: } & \text { Municipal Solid Waste } \\ \text { VRP: } & \text { Vehicle Routing Problem } \\ \text { CVRP: } & \text { Capacitated Vehicle Routing Problem } \\ \text { SHS } & \text { Standard Harmony Search } \\ \text { IHHS: } & \text { Intelligence Hybrid Harmony Search }\end{array}$

\section{Acknowledgements}

The authors would like to thank Faculty of Interdisciplinary Studies, Khon Kaen University, Nong Khai Campus for supporting this research.

\section{Funding}

This study was funded by Research and Academic Services Transfer Affairs of Khon Kaen University.

Availability of data and materials

In this paper, all of datasets are derived from the VRP Web (http://www.bernabe.dorronsoro.es/vrp/).

Competing interests

The authors declare that they have no competing interests.

Authors' contributions

Wullapa Wongsinlatam designed the mathematical mo del, the new metaheuristic algorithm, and the computational framework. Ayuwat Thanasate-angkool collected and analysed the data. Wullapa Wongsinlatam carried out the experimental of the model. Wullapa Wongsinlatam and Ayuwat Thanasate-angkool wrote the manuscript with input from all authors.

\section{Author details}

${ }^{1}$ Faculty of Interdisciplinary Studies, Khon Kaen University, Nong Khai Campus, Nong Khai,43000, Thailand. ${ }^{2}$.

References

1. Badran MF, El-Haggar SM. Optimization of municipal solid waste management in Port Said-Egypt. Waste Manag. 2006;26:534-545.

2. Li F, Cai B, Ye Z, Wang Z, Zhang W, Zhou P, Chen J. Changing patterns and determinants of transportation carbon emissions in Chinese cities. Energy. 2019;174:562-575.

3. Heidari R, Yazdanparast R, Jabbarzadeh A. Sustainable design of a municipal solid waste management system considering waste separators: A real-world application. Sustain. Cities Soc. 2019;47: 101457

4. loan-Robert I, Diego I, José-Luis G-M, Javier D. Review of life-cycle environmental consequences of waste-to-energy solutions on the municipal solid waste management system. Resources, Conservation and Recycling. 2020; https://doi.org/10.1016/j.resconrec.2020.104778

5. Doaemo W, Dhiman S, Borovskis A, et al. Assessment of municipal solid waste management system in Lae City, Papua New Guinea in the context of sustainable development. Environ Dev Sustain. 2021; https://doi.org/10.1007/s10668-021-01465-2

6. Batur ME, Cihan A, Korucu MK, Bektas N, Keskinler B. A mixed integer linear programming model for long-term planning of municipal solid waste management systems: Against restricted mass balances. Waste Manag. 2020. 105:211-222.

7. Sala S. Chapter 3-Triple bottom line, sustainability and sustainability assessment, an overview. In Biofuels for a More Sustainable Future. Elsevier: Amsterdam. 2020. doi: 10.1016/B978-0-12-815581-3.00003-8.

8. Maria C, Góis J, Leitão A. Challenges and perspectives of greenhouse gases emissions from municipal solid waste management in Angola. Energy Rep. 2019; https://doi.org/10.1016/j.egyr.2019.08.074

9. Mohsenizadeh M, Tural MK, Kentel E. Municipal solid waste management with cost minimization and emission control objectives: A case study of Ankara. Sustain. Cities Soc. 2020; https://doi.org/10.1016/j.scs.2019.101807

10. Tang CS, Zhou S. Research advances in environmentally and socially sustainable operations. Eur. J. Oper. Res. 2012;223:585-594.

11. Li J-Q, Borenstein D, Mirchandani PB. Truck scheduling for solid waste collection in the City of Porto Alegre, Brazil. Omega. 2008;36: 1133-1149. 
12. Paolo T, Daniele V. Vehicle Routing: Problems, Methods, and Applications, Second Edition. Soc. Ind. Appl. Math. 2014. https://doi.org/10.1137/1.9781611973594

13. Lawler EL, Wood DE. Branch-and-bound methods: A survey Oper. Res. 1966. https://doi.org/10.1287/opre.14.4.699

14. Ralph EG. Outline of an algorithm for integer solutions to linear programs and an algorithm for the mixed integer problem. Springer, Berlin, Heidelberg. 2010;77-103

15. Cynthia B, Ellis Lj, George L N, Martin WPS, Pamela HV, Branch-and-price: Column generation for solving huge integer programs Oper. Res. 1998;46:3: 316-329

16. Viotti P, Polettini A, Pomi R, Innocenti C. Genetic algorithms as a promising tool for optimisation of the MSW collection routes. Waste Manag. Res. 2003. doi: 10.1177/0734242X0302100402

17. Hannan MA, Akhtar M, Begum RA, Basri H, Hussain A, Scavino E. Capacitated vehicle routing problem model for scheduled solid waste collection and route optimization using PSO algorithm. Waste Manag. 2018;71:31-41.

18. Kennedy J, Eberhart RC, Particle swarm optimization. IEEE Transaction. 1995;4:1942-7.

19. Dorigo M, Gambardella LM, Ant Colony System: A cooperative learning approach to the traveling salesman problem. IEEE Transactions on Evolutionary Computation. 1997;1:1:53-66.

20. Charbel R, Jean-Paul A, ACO for the Surgical Cases Assignment Problem. Journal of Medical Systems. 2012;36: 1891-1899.

21. Xin SY, Suash D, Cuckoo search via Lvy flights. Mathematical Mod- eling and Numerical Optimization. 2010;1:4:210-215.

22. Yang XS, Firefly algorithm, In Nature-Inspired Metaheuristic Algorithms. Ed., Wiley Online Library: Hoboken. NJ, USA. 2008;7990.

23. Yang XS, Firefly algorithm, Levy flights and global optimization. In Research and Development in Intelligent Systems. XXVI; Springer: New York, NY, USA. 2010;209218.

24. Wongsinlatam W, Buchitchon S. The comparison between Dragonflies Algorithm and Fireflies Algorithm for Court Case Administration: A Mixed Integer Linear Programming. Journal of Physics: Conference Series. 2018; doi:10.1088/1742-6596/1061/1/012005.

25. Geem ZW, Kim JH, Loganathan GV. A new heuristic optimization algorithm: harmony search. Simulation. 2001;76:2:60-68.

26. Yassen ET, Ayob M, Nazri MZ, Sabar NR. Meta-harmony search algorithm for the vehicle routing problem with time windows. Information Sciences. 2001;325:140-158

27. Maleki F, Yousekhoshbakht M, Rahati A. A hybrid self-adaptive global best harmony search algorithm for the vehicle routing problem with time windows. BRAIN. Broad Research in Articial Intelligence and Neuroscience, 2017;8:4::65-84.

28. Mahima D, Vijay K, Manjit K, Thanh-Phong D. A Systematic Review on Harmony Search Algorithm: Theory, Literature, and Applications. Mathematical Problems in Engineering. 2021. doi.org/10.1155/2021/5594267

29. Liu J, He Y, A clustering-based multiple ant colony system for the waste collection vehicle routing problems. In Proceedings of the 2012 Fifth International Symposium on Computational Intelligence and Design, Hangzhou, China, 28-29 October 2012.

30. Kuo RJ, Zulvia FE, Suryadi K. Hybrid particle swarm optimization with genetic algorithm for solving capacitated vehicle routing problem with fuzzy demand-A case study on garbage collection system. Appl. Math. Comput. 2012; 219:2574-2588

31. Akhtar M, Hannan MA, Begum RA, Basri H, Scavino E. Backtracking search algorithm in CVRP models for effcient solid waste collection and route optimization. Waste Manag. 2017;61:117-128. 
Figures

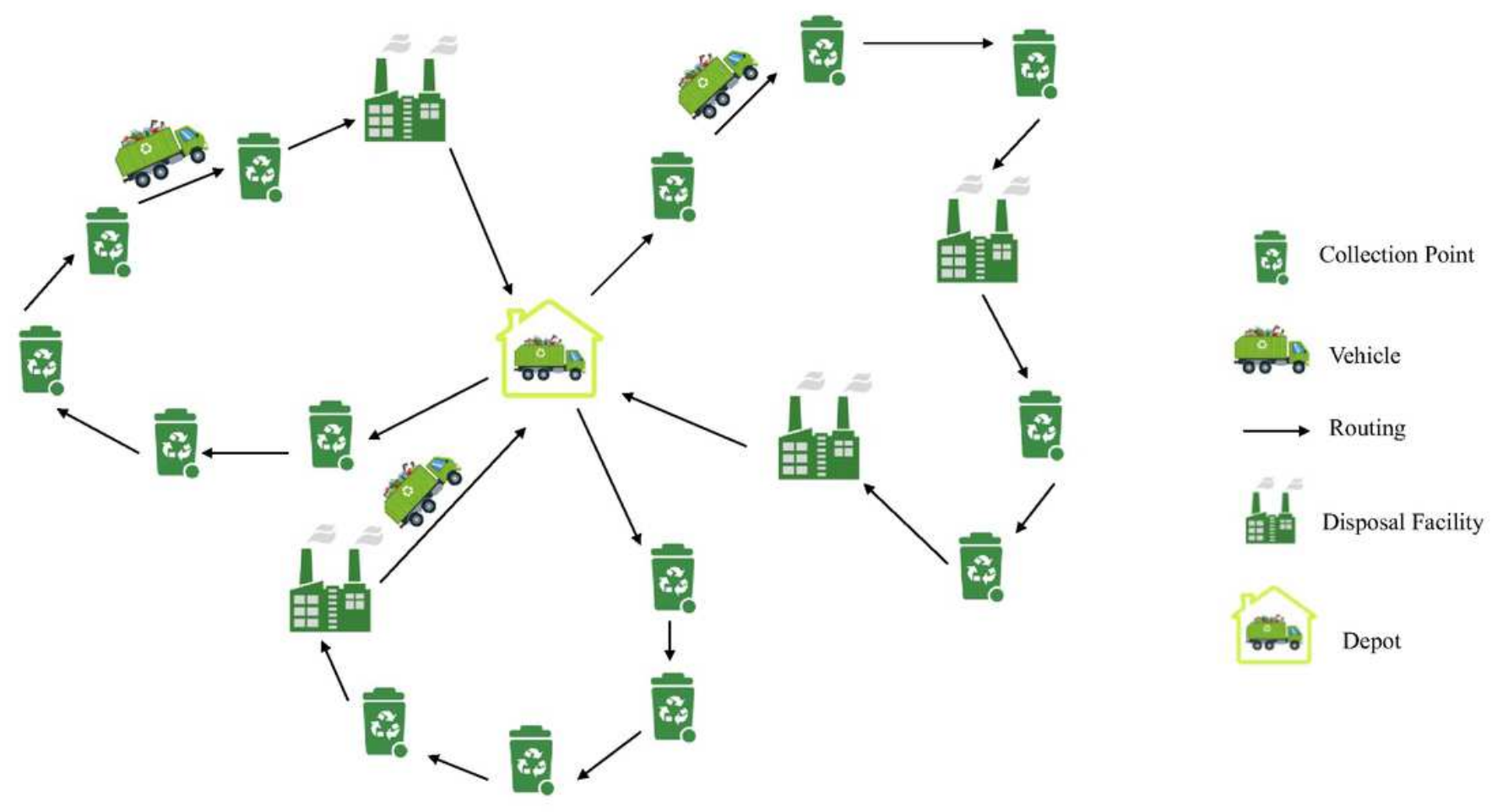

Figure 1

The diagram of the MSW collection 

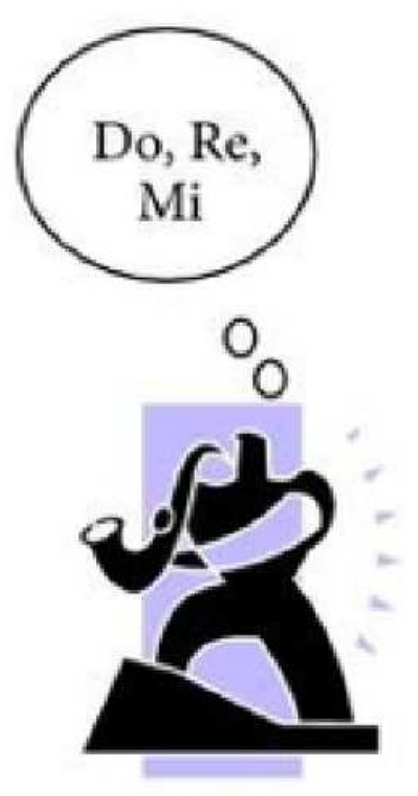

$$
=\mathrm{Do}
$$

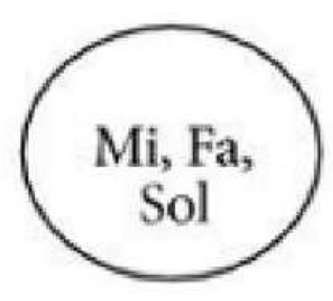

$\mathrm{O}$

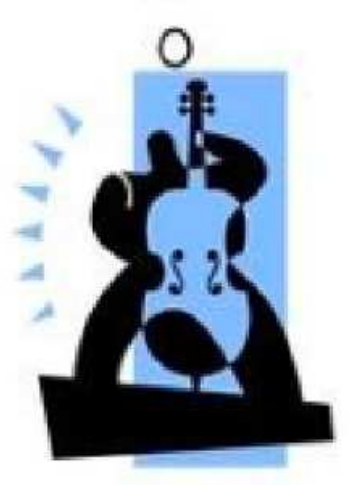

$=\mathrm{Mi}$
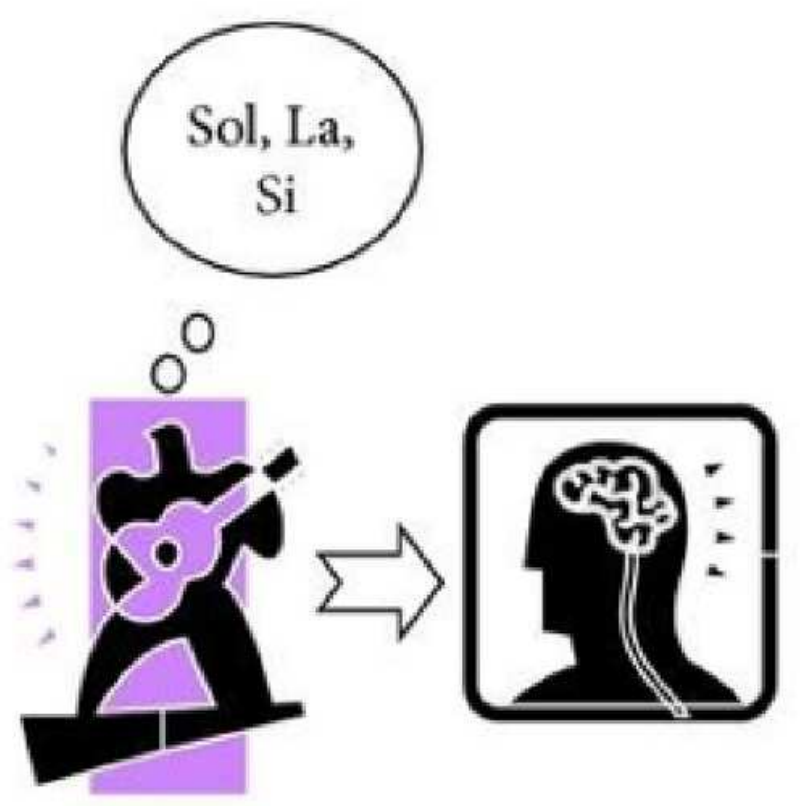

$=$ Sol

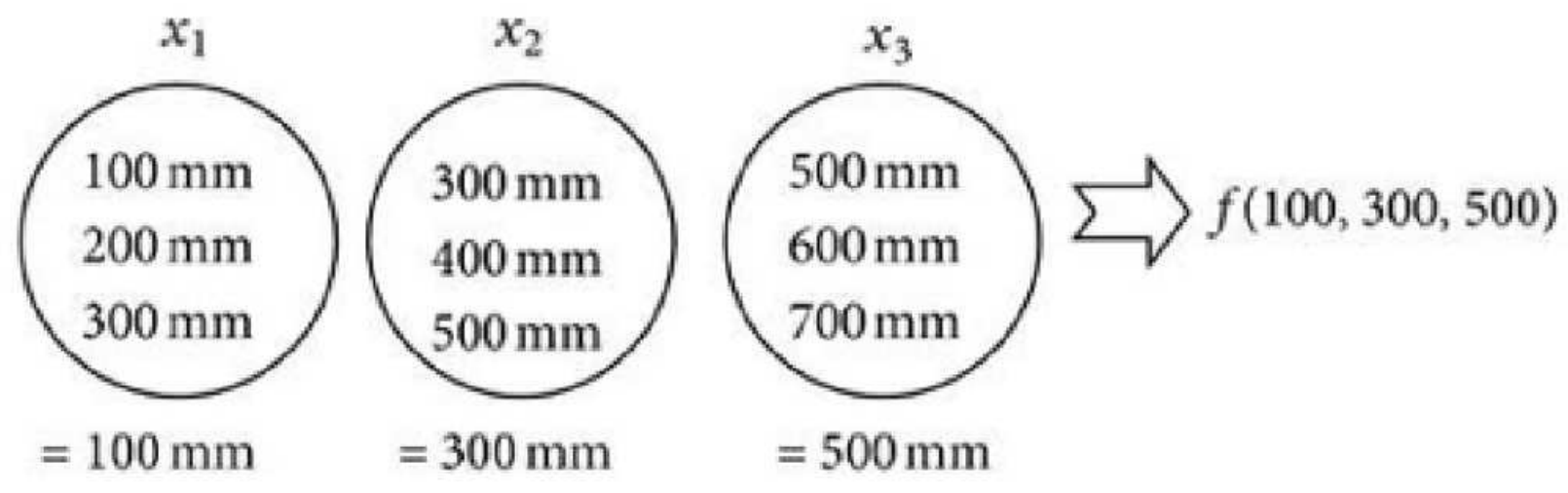

Figure 2

Analogy between music improvisation and optimization problem (Geem et al., 2001) 